\title{
artigo
}

Nascimento, F.J.; Ferreira Silva, D.R.; Barbosa, H.C.; Santos, V.F.; Martins, L.M.; Luz, D.C.R.P.;

Sobrepeso e obesidade em adolescentes escolares: uma revisão sistemática

\section{Sobrepeso e obesidade em adolescentes escolares: uma revisão sistemática}

\author{
Overweight and obesity in school adolescents: a systematic review \\ El sobrepeso y la obesidad en los adolescentes escolares: una revisión sistemática
}

\begin{abstract}
RESUMO
O objetivo do estudo foi analisar a prevalência de sobrepeso e obesidade em adolescentes escolares. Trata-se de uma revisão sistemática realizada no banco de dados BDENF, Lilacs e SciELO, onde foram analisados 08 trabalhos, nos idiomas português e espanhol no período de 2017 a 2019, na área temática da saúde e educação. Os resultados apontaram para um quadro de obesidade e sobrepeso de parcela significativa dos escolares investigados, firmando a relação entre esta conjuntura e a prevalência e/ou desencadeamento das doenças crônicas não transmissíveis, sendo mais presente naqueles que frequentam os ambientes educacionais da rede pública. Assim sendo, uma vez que o ambiente escolar é um espaço privilegiado para realização de levantamento de dados e programas de intervenções em saúde, aponta-se para a necessidade de medidas preventivas e de promoção à saúde, que visem atender os indivíduos e a família de forma integral e contínua, por meio de ações multissetoriais. DESCRITORES: Sobrepeso; Obesidade; Adolescentes; Estudantes.
\end{abstract}

\section{ABSTRACT}

The objective of the study was to analyze the prevalence of overweight and obesity in school adolescents. It is a systematic review conducted in the BDENF, Lilacs and SciELO database, where 08 papers were analyzed, in Portuguese and Spanish languages from 2017 to 2019, in the thematic area of health and education. The results pointed to a picture of obesity and overweight of a significant portion of the schoolchildren surveyed, establishing the relationship between this situation and the prevalence and/or triggering of chronic non-communicable diseases, being more present in those who attend the educational environments of the public network. Thus, since the school environment is a privileged space for data collection and health intervention programs, it points to the need for preventive and health promotion measures, which aim to serve individuals and the family in an integral and continuous manner, through multisectoral actions.

DESCRIPTORS: Overweight; Obesity; Adolescent; Students.

\section{RESUMEN}

El objetivo del estudio era analizar la prevalencia del sobrepeso y la obesidad en los adolescentes de las escuelas. Se trata de una revisión sistemática realizada en la base de datos del BDENF, Lilacs y SciELO, donde se analizaron 08 trabajos, en idiomas portugués y español de 2017 a 2019, en el área temática de la salud y la educación. Los resultados señalaron un cuadro de obesidad y sobrepeso de una parte importante de los escolares encuestados, estableciendo la relación entre esta situación y la prevalencia y/o el desencadenamiento de enfermedades crónicas no transmisibles, estando más presente en los que asisten a los entornos educativos de la red pública. Así pues, dado que el entorno escolar es un espacio privilegiado para la recopilación de datos y los programas de intervención en materia de salud, señala la necesidad de adoptar medidas preventivas y de promoción de la salud, que tengan por objeto servir a los individuos y a la familia de manera integral y continua, mediante acciones multisectoriales. DESCRIPTORES: Overweight; Obesidad; Adolescentes; Estudiantes.

RECEBIDO EM: 17/07/2020 APROVADO EM: 20/07/2020

\section{Francisco Junio do Nascimento}

Enfermeiro, Pós Graduando em Docência do Ensino Superior com Ênfase em Sistema de Saúde - Faculdade de Venda Nova do Imigrante / FAVENI.

ORCID: 0000-0002-5010-9747 


\title{
Débora Rayanne Ferreira Silva
}

Enfermeira. Graduada em Enfermagem pela Faculdade de Juazeiro do Norte - FJN (2020). Enfermeira Assistencialista no Hospital de Campanha Covid-19, Juazeiro do Norte-CE. Tem experiência na área de Enfermagem.

ORCID: 0000-0003-1681-4116

\section{Hellen Cavalcanti Barbosa}

Enfermeira. Graduada em Enfermagem pela Faculdade de Juazeiro do Norte - FJN (2020). Enfermeira Assistencialista na Estratégia Saúde da Família (ESF), Remanso, Orocó-PE. Tem experiência na área de Enfermagem.

ORCID: 0000-0003-0579-3903

\author{
Viviane Ferreira dos Santos \\ Graduanda em Enfermagem - Faculdade de Juazeiro do Norte / FJN. \\ ORCID: 0000-0002-0289-6627
}

\section{Lucielton Mascarenhas Martins}

Educador Físico, Mestre em Educação - UPE campus Petrolina. Docente do Instituto Federal de Educação, Ciência e Tecnologia da Bahia campus Seabra.

ORCID: 0000-0001-7743-0859

\section{Dayse Christina Rodrigues Pereira Luz}

Enfermeira, Pós Doutoranda em Ciências da Saúde - Centro Universitário Saúde ABC / FMABC. Docente da Faculdade de Juazeiro do Norte - FJN.

ORCID: 0000-0002-5719-3574

\section{INTRODUÇÃO}

$\mathbf{N}$ a contemporaneidade, o cenário mundial é marcado por um crescente índice da prevalência de obesidade e sobrepeso, que pode ser observado nos mais diversos países, independente do nível de desenvolvimento socioeconômico, mesmo não descartando a possibilidade de relação entre este fator, juntamente com indicativos hereditários, de estilo de vida e ao processo de ordem tecnológico ${ }^{(1,2)}$.

Transformando-se na desordem nutricional que mais acendeu no mundo, a obesidade e o sobrepeso, sobressaem-se como uma das condições mais prevalentes e um dos principais problemas de saúde pública, pelas consequências fisiológicas, prejuízos cognitivos e comportamentais e efeitos deletérios à qualidade de vida ${ }^{(3)}$. Os fatores de risco têm sido creditados às transformações sociais, culturais, ambientais e comportamentais, expressos nas mudanças no padrão alimentar e estilo de vida.

A Organização Mundial da Saúde OMS define obesidade como uma patologia caracterizada pelo acúmulo anormal ou excessivo de gordura no organismo, resultando na perda da qualidade de vida
Transformando-se na desordem nutricional que mais acendeu no mundo, a obesidade

e o sobrepeso, sobressaem-se como uma das condições mais prevalentes $\mathrm{e}$ um dos principais problemas de saúde pública e diminuindo a longevidade, que resulta do desequilíbrio crônico entre o consumo alimentar e o gasto energético ${ }^{(4)}$.

Este panorama apresenta etiologia multifatorial, com fatores biológicos, metabólicos, socioculturais, comportamentais e ambientais, assim, têm sido alvos de diversas investigações científicas, principalmente pelo fato de na adolescência existir grande probabilidade de aumento destes casos pelas alterações metabólicas ${ }^{(5)}$ e devido à tese de que a duração e a gravidade da obesidade nesta fase determinam o nível das alterações metabólicas que são estendidas até a fase adulta.

$\mathrm{Na}$ adolescência o crescimento, desenvolvimento e nutrição são aspectos essenciais e interdependente. Esta fase da vida é analisada, de maneira especial, como vulnerável, em aspectos nutricionais, devido a uma maior carência geral e necessidades especiais de nutrientes, instigadas pela velocidade de crescimento e pelas alterações do estilo de vida e dos hábitos alimentares assumidos ${ }^{(6)}$.

Os limites cronológicos da adolescência são definidos pela OMS entre 10 e 19 anos, e no Brasil, o Estatuto da Criança e do Adolescente, Lei no 8.069, de 1990, considera adolescência como a faixa etária de 12 a 18 anos de idade (art. 2o $)^{(7)}$. 


\section{artigo}

$\mathrm{Na}$ adolescência os indivíduos experimentam mudanças biológicas, cognitivas, emocionais e sociais, e vivenciam um importante momento para a adoção de novas práticas, uma vez que, os hábitos adquiridos nessa etapa podem persistir por toda vida, e o início precoce de comportamentos maléficos concebe risco para o surgimento de doenças crônicas não transmissíveis ${ }^{(8,9)}$. Podemos considerar a obesidade e sobrepeso como a nova síndrome mundial, no Brasil, bem como nos países desenvolvidos, uma vez que há uma crescente progressão no que se relaciona a transição nutricional e ao excesso de peso em escolares ${ }^{(10)}$.

Existem vários procedimentos para o controle da obesidade e do sobrepeso, dependendo da causa e da gravidade da sua condição, destacando que estes procedimentos devem ser acompanhados por profissionais da área ${ }^{(11)}$. É compreensível que os profissionais da área da saúde conservem seus conhecimentos atualizados em relação às consequências, causas e formas de tratamento do sobrepeso e obesidade, bem como os componentes utilizados.
Nos ambientes escolares temos a necessidade e preocupação sobre o tema saúde, e destacamos o Programa Saúde na Escola - PSE como ferramenta primordial na prevenção deste quadro relativo à obesidade e sobrepeso, na medida em que a evolução política e social influenciaram a apropriação da escola como espaço de promoção à saúde. O programa objetiva à sensibilização e mudança de hábitos, contribuindo para a formação integral dos estudantes, da rede pública de ensino, por meio de ações de promoção, prevenção e atenção à saúde $e^{(12,13)}$.

Assim, notando-se a necessidade de criar mecanismos de orientação às ações dos serviços de saúde, e seus respectivos profissionais, para atender às necessidades e demandas da população, o referido estudo tem como objetivo analisar a prevalência de sobrepeso e obesidade em adolescentes escolares.

\section{METÓdO}

Trata-se de um estudo do tipo revisão sistemática, realizada durante os meses de

\section{Figura 01: Fluxograma do processo de seleção dos artigos pesquisados. Juazeiro do Norte, CE, Brasil, 2020.}

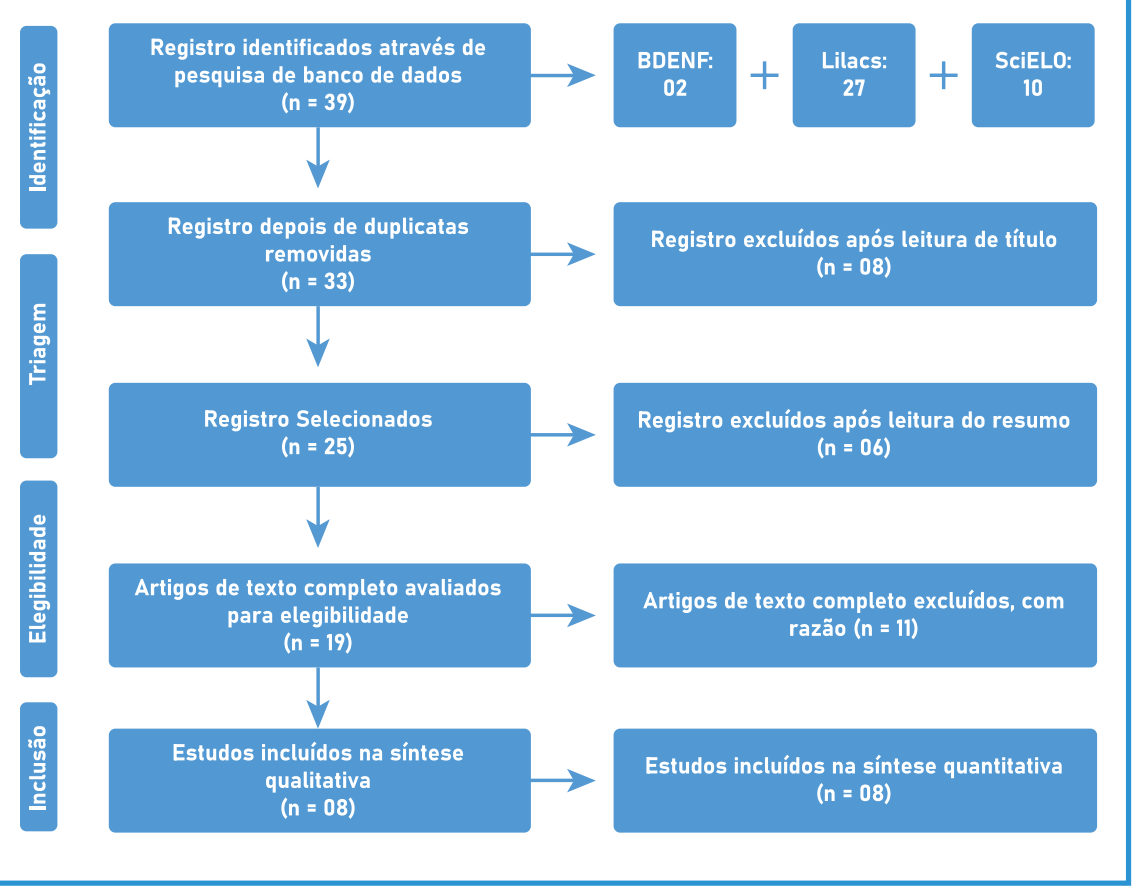

abril e maio de 2020. Os artigos analisados são oriundos das bases de dados eletrônicas BDENF, Lilacs e SciELO, nos idiomas português e espanhol no período de 2017 a 2019, na área temática da saúde, educação e seus desdobramentos. A busca foi executada com base nos Descritores em Ciências da Saúde - DeCS, utilizando os seguintes termos "Sobrepeso", "Obesidade", "Adolescentes" e "Estudantes" com operador booleano "and".

Como critérios de inclusão utilizou-se todos os artigos originais indexados na versão completa e gratuita, com caracterização de estudos de casos, observacionais, documentais e realizados no Brasil. Foram excluídos artigos duplicados, aqueles sem ideia central pautada na temática abordada, na área empresarial e que não se enquadrassem nas modalidades de saúde e educação e aqueles na modalidade de revisão sistemática e/ou integrativa.

Os artigos foram submetidos às seguintes etapas de avaliação, com base no protocolo Prisma (Preferred Reporting Items for Systematic Rewiews and Meta-Analisys): Identificação dos Registros através de pesquisa nos bancos de dados; Triagem por meio da exclusão de arquivos duplicados e daqueles que não contemplavam os critérios de inclusão por meio da leitura dos títulos e resumos; Elegibilidade através da leitura do artigo na integra; e Inclusão dos trabalhos que se adequaram nos critérios e objetivos da referida pesquisa.

Para análise dos materiais selecionados, teve-se o problema refletido na seguinte pergunta, Qual a prevalência de sobrepeso e obesidade em adolescentes escolares?, de acordo com o acrônimo PICo, onde $\mathrm{P}=$ adolescentes, $\mathrm{I}=$ prevalência e Co $=$ prevalência de sobrepeso e obesidade.

\section{RESULTADOS}

$\mathrm{Na}$ Figura 01 apresentamos os resultados encontrados, de acordo com o fluxograma do processo de revisão. Em sequência, na Tabela 01, apresenta-se um panorama geral dos artigos analisados.

Após processo de seleção do material, foram restauradas 33 referências após a 
pesquisa com o cruzamento dos temas geradores no banco de dados e eliminação dos materiais duplicados, após esta remoção, por meio da leitura dos títulos, foram eleitos 25 trabalhos para consulta dos resumos, onde estiveram selecionados 19 trabalhos para leitura na integra e após a leitura na integral para avaliação dos métodos e resultados foram seletos 08 trabalhos.

\section{DISCUSSÕES}

A partir da análise dos dados foi perceptível que parcela significativa dos estudos abordam, além dos adolescentes,

Tabela 01: Perfil dos dados obtidos dos artigos analisados. Juazeiro do Norte, CE, Brasil, 2020.

Autor Título Objetivo

Dalmaso et al..144)

Prevalência de sobrepeso e obesidade em escolares do ensino fundamental de Vitória/ES

Eid et al. ${ }^{(15)}$

Teixeira et al. (16)

Mendonça et al. ${ }^{(17)}$

Avaliação dos níveis pressóricos e antropométricos de escolares no interior do nordeste brasileiro

Estado nutricional de escolares

Conde et al. ${ }^{(18)}$

Mundstock et al. ${ }^{(19)}$

Resultados da primeira fase do Programa Esporte e Saúde em Canela, Rio Grande do Sul: avaliação do perfil nutricional

Pereira et al. (20)

Fatores de risco e proteção contra doenças crônicas não transmissiveis entre adolescentes

Estado nutricional dos alunos da Rede Nacional de Ensino de Anjos; Silveira(21)
Investigar a prevalência de sobrepeso e obesidade em crianças do ensino fundamental de Vitória/ ES.

Avaliar os hábitos alimentares e a frequência dos seguintes fatores de risco para hipertensão arterial em escolares: obesidade, sobrepeso, obesidade abdominal, inatividade física, antecedentes familiares e níveis pressóricos elevados.

Identificar estado nutricional relacionado à imagem corporal e ao risco cardiovascular de adolescentes das escolas pública e privada.

Avaliar a influência do excesso de peso nos valores de pressão arterial de escolares 4 a 17 anos em uma cidade no interior de Sergipe.

Descrever dados Antropométricos da Pesquisa Nacional de Saúde dos Escolares (PeNSE) 2015 e sua distribuição segundo estratos geográficos e socioeconômicos.

Apresentar os resultados da avaliação nutricional de crianças e adolescentes, como parte de um programa municipal voltado à saúde de escolares.

Conhecer a prevalência dos fatores de risco para doenças crônicas não transmissíveis entre adolescentes assistidos pelo programa municipal Saúde e Prevenção na Escola - SPE.

Identificar e descrever o crescimento e o perfil nutricional antropométrico das crianças matriculadas na Rede Nacional de Ensino do Sesc.

\section{Resultados}

A alta prevalência de sobrepeso e obesidade reafirma que o excesso de peso atinge crianças e adolescentes que frequentam escolas municipais significativamente.

Evidencia a exposição de escolares a múltiplos fatores de risco para doenças crônicas e podem nortear ações intersetoriais de comunidades acadêmicas, autoridades educacionais e de saúde.

Independente do estado nutricional há insatisfação da imagem corporal entre os adolescentes e o risco cardiovascular está associado ao sobrepeso e obesidade.

Crianças e adolescentes com pressão arterial alterada exibem maior média de IMC, confirmando a necessidade de intervenção para prevenção e promoção da saúde.

0 excesso de peso é mais frequente entre adolescentes dos estratos de baixa renda, indicando, além do estado nutricional, desigualdade social no Brasil.

Os estudantes apresenta expressivo índice de sobrepeso/obesidade, reforçando a necessidade de programas de prevenção e tratamento da obesidade, estimulando a prática de atividade física e alimentação saudável.

A população apresenta distintos padrões de exposição a fatores de risco e proteção, e evidencia a necessidade de ações educativas no ambiente escolar conduzidas pelos profissionais do SPE e demais gestores escolares.

A prevalência de excesso de massa corporal para crianças acima de 10 anos foi de $37,4 \%$, sendo necessário o acompanhamento do estado nutricional devido aos agravos e efeitos na vida adulta. 
as crianças, o que nos remete a finalidade de identificação e prevenção deste quadro nutricional em suas sinalizações mais incipientes, para um maior controle, conscientização e prevenção, evitando futuramente culminâncias mais agudas e o risco de acometimento de doenças crônicas não transmissíveis, visto que enquanto mais cedo a obesidade se desenvolve, maiores são as chances da incidência na vida adulta ${ }^{(14,17,18,22)}$.

Os métodos de pesquisa, em sua totalidade, isentaram-se de intervenções e acompanhamentos em caráter longitudinal, método este que poderia nos aproximar de instrumentos/meios, de aplicação prática, para lidar com este quadro especifico da obesidade e sobrepeso. Diversos fatores na contemporaneidade favorecem para uma crescente taxa de obesidade em idade jovem, sendo válido ressaltar a influência dos hábitos parentais, do ambiente doméstico e do desenvolvimento tecnológico ${ }^{(15)}$.

Aponta-se a necessidade de abordagens preventivas, programas de educação em saúde e políticas públicas multidisciplinares eficazes para o controle dessas condições, visto os fatores multifatoriais, com ênfase nos hábitos alimentares saudáveis e a prática regular de atividade física $^{(14,16,19)}$, sendo inestimável que os profissionais da saúde direcionem um olhar reflexivo para o panorama e o considerem uma oportunidade de implementação de medidas de prevenção e acompanhamento de caráter mutissetorial ${ }^{(15)}$.

Em sua totalidade, os resultados apontaram para o quadro de obesidade e sobrepeso de parcela significativa dos escolares investigados, firmando, também, a relação entre esta conjuntura e a prevalência e/ou desencadeamento das doenças crônicas não transmissíveis, independente das condições econômicas, mas sendo mais presente naqueles que frequenta os ambientes educacionais da rede pública ${ }^{(18)}$.

A obesidade e o sobrepeso, em suma, trazem comprometimento não só à saúde, mas à qualidade de vida e ao impacto econômico, em especial pela vulnerabilidade dos adolescentes ${ }^{(17,19,20)}$, podemos considerar este quadro como uma pandemia ${ }^{(17)}$.
Segundo a OMS, mais de 340 milhões de crianças e adolescentes apresentaram sobrepeso e obesidade em 2016, tendo um aumento de $14 \%$ quando comparado aos dados de 1975 para a mesma coleta com indivíduos de 5 a 19 anos ${ }^{(14)}$.

No Brasil, bem como os países em desenvolvimento, vem apresentando uma evolução do excesso de peso de sua população, esse fator deve-se aos novos padrões de vida e hábitos associados ao desenvolvimento social e tecnológico, favorecendo a inatividade física que é fator contribuinte para o sedentarismo, e ao processo de ingestão de alimentos ricos em açúcares e gorduras ${ }^{(23,24)}$.

Reafirmamos a tese de que a obesidade é uma doença crônica multifatorial não transmissível e representa um dos principais problemas de saúde pública em países desenvolvidos e em desenvolvimento ${ }^{(14,16,17,21)}$, sendo que a sua expansão segue ritmo mais rápido para os mais pobres, acentuando as desigualdades ${ }^{(18)}$. $\mathrm{O}$ excesso de peso afeta uma proporção significativa de escolares e a idade apresenta efeito direto sobre o peso corporal, esse índice reforça a recomendação e necessidade de ações preventivas ao desenvolvimento da obesidade e sobrepeso durante a juventude, fase de transformações físicas, cognitivas, emocionais e sociais $^{(15,16,21)}$.

Um outro aspecto que merece destaque é a instauração da obesidade e sobrepeso na população jovem independente do sexo, uma vez que alguns estudos destacam que as meninas apresentaram proporções maiores, mas mantendo valores proximais ${ }^{(14,18)}$. Contudo, alguns outros estudos apontam que as maiores médias de acúmulo de gordura foram observadas em indivíduos do sexo masculino ${ }^{(15,20)}$. Em outros casos os dados foram estatisticamente semelhantes para os $\operatorname{sexos}^{(17,21)}$.

Os dados direcionam, não obstante das patologias, como risco cardiovascular e a hipertensão arterial ${ }^{(14)}$, para uma insatisfação da imagem corporal dos adolescentes, pautada em padrões sociais, quando acometidos pelo excesso de peso, independente de classe social e da instituição de ensino (pública ou privada) ${ }^{(16)}$. Um dos estudos destaca a preocupação de que possa ser desencadeado transtornos alimentares e/ou dietas inapropriadas, sem orientação profissional, em meio a busca pela autoestima corporal e a indispensável relação da escola na conscientização quanto a educação alimentar e no combate a obesidade, reforçando ainda que, o excesso de peso em escolares ofertam, para estes, maior risco de desenvolvimento de problemas de saúde de ordem cardiometabólica ${ }^{(17)}$.

Destaca-se o Plano de Ações Estratégicas para o Enfrentamento das Doenças Crônicas Não Transmissíveis 2011-2022, traçada pelo Brasil, com o intuito de promover o desenvolvimento e a implementação de políticas públicas efetivas, com base em evidências. Neste contexto o Projeto Saúde e Prevenção nas Escolas, integrante deste plano, tendo como finalidade contribuir de maneira intensificada na formação integral dos escolares da rede pública de educação básica, através de ações de prevenção, promoção e atenção à saúde(20). Destaca-se o ambiente escolar como espaço privilegiado para realização de levantamento de dados e programas de intervenções em saúde ${ }^{(21)}$.

As pesquisas analisadas apontam para limitações relativas: ao número amostral analisado, destacando a necessidade de extrapolação de uma análise a nível nacional; aos métodos de coleta de dados, onde os valores de referências podem não ser relativamente contextuais; à transversalidade dos estudos ${ }^{(17,18)}$.

\section{CONCLUSÃO}

É possível afirmar que o aumento significativo nas prevalências de obesidade em adolescentes tem se tornado irrefutável, apontando para a necessidade de medidas preventivas, uma vez que este grupo está diretamente exposto à ambientes e hábitos favoráveis. Assim, a prevenção, comparada com o tratamento, por meio de intervenções em fases precoces, ainda é a maneira mais eficaz de combate a esta doença, intercessões essas capazes de relacionar o ambiente familiar, social e educacional dos adolescentes.

Diante do contexto, é necessário à 
implementação de medidas não só de prevenção, mas, também, de promoção à saúde mais abrangentes, talvez a atenção primária da saúde e o ambiente escolar, que visa atender os indivíduos e a família de forma integral e contínua, seja o espaço inicial para este processo de promoção à saúde, uma vez que estes profissionais têm frequentemente a oportunidade de atuar aconselhando sobre nutrição, atividade física e esclarecendo dúvidas sobre doenças crônicas não transmissíveis. .

\section{REFERÊNCIAS}

1. Moura JRA, Silva KCB, Rocha AESH, Santos SD, Amorim TRS, Silva ARV. Construção e validação de cartilha para prevenção do excesso ponderal em adolescentes. Acta Paul. Enferm. 2019; 32 (4): 365-373.

2. Alcântara CM, Silva ANS, Pinheiro PNC, Queiroz MVO. Tecnologias digitais para promoção de hábitos alimentares saudáveis dos adolescentes. Rev. Bras. Enferm. 2019; 72 (2): 513-520.

3. Pinto RP, Nunes AA, Mello LM. Análise dos fatores associados ao excesso de peso em escolares. Rev. Paul. Pediatr. 2016; 34 (4): 460-468.

4. Bellodi, AC. Obesidade em crianças e adolescentes: temperamento, estresse, coping e risco psicossocial familiar. 2018. 221p. Tese (Programa de Pós-Graduação em Psicologia) - Pontifícia Universidade Católica de Campinas, Campinas-SP.

5. Sales-Peres SHC, Goya S, Sant'Anna RMF, Silva HM, Sales-Peres AC, Silva RPR et al. Prevalência de sobrepeso e obesidade e fatores associados em adolescentes na região centro-oeste do estado de São Paulo (SP, Brasil). Ciênc. saúde coletiva. 2010; 15 (Supl 2): 3175-3184.

6. Pereira AMGR. Preocupação com o peso e prática de dietas por adolescentes. Acta Port Nutr. 2016; (6): 14-18.

7. Brasil. Ministério da Saúde. Secretaria de Atenção à Saúde. Departamento de Ações Programáticas e Estratégicas. Proteger e cuidar da saúde de adolescentes na atenção básica. 2. ed. Brasília: Ministério da Saúde, 2018.

8. Carneiro CS, Peixoto MRG, Mendonça KL, Póvoa TIR, Nascente FMN, Jardim TSV et al. Excesso de peso e fatores associados em adolescentes de uma capital brasileira. Revista Brasileira de Epidemiologia. 2017; 20 (2): 260-273.

9. Carvalho MSN. Prevalência e fatores de risco associados ao sobrepeso e obesidade em adolescentes da cidade de São Luís, Maranhão. 2016. 100p. Dissertação (Programa de Pós-Graduação em Saúde do Adulto e da Criança) - Universidade Federal do Maranhão. São Luís.

10. Tornquist L, Tornquist D, Franke SIR, Burgos MS. Programas de intervenção com escolares obesos no Brasil: uma revisão na base de dados do Scientific Electronic Library Online. Revista Baiana de Saúde Pública. 2016. 40 (4): 832-847.

11. Fernandes DD. Dislipidemia e obesidade central em adultos jovens escolares. 2017. 69p. Dissertação (Programa de Pós-Graduação em Saúde Coletiva) - Universidade Estadual do Ceará. Fortaleza, Ceará.

12. Vieira CENK, Dantas DNA, Miranda LSMV, Araújo AKC, Monteiro Al, Enders BC. Programa de Enfermagem Saúde na Escola: prevenção e controle de sobrepeso/obesidade em adolescentes. Rev. esc. enferm. USP. 2018; 52.
13. Vieira LS, Saporetti GM, Belisário SA. Programa saúde na escola: marcos jurídicos e institucionais. Rev. Med. Minas Gerais. 2016; 26 (Supl 8): 381-387.

14. Dalmaso SB, Sant'ana PG, Cordeiro JP, Rodrigues AL, Ferreira LG, Leopoldo AS et al. Prevalência de sobrepeso e obesidade em escolares do ensino fundamental de Vitória/ES. O Mundo Saúde. 2019; 43 (1): 61-82.

15. Eid LP, Barroso NNN, Kruk LBL, Lima APA, Pompeo DA, Ribeiro SAB. Hábitos alimentares e fatores de risco para hipertensão arterial sistêmica em escolares. Archives of Health Sciences. 2019; 26 (1): 9-14.

16. Teixeira CS, Dinardi B, Singh TC, Cesarino CB, Pompeo, DA. Estado nutricional de adolescentes relacionado ao risco cardiovascular e imagem corporal. O Mundo saúde. 2019; 43 (1): 249264.

17. Mendonça RG, Ribeiro BVS, Oliveira LL, Nunes TS, Tanajura DM. Avaliação dos níveis pressóricos e antropométricos de escolares no interior do nordeste brasileiro. Revista Brasileira de Ciência da Saúde. 2019; 23 (4): 439-448.

18. Conde WL, Mazzeti CMS, Silva JC, Santos IKS, Santos AMR. Estado nutricional de escolares adolescentes no Brasil: a Pesquisa Nacional de Saúde dos Escolares 2015. Revista Brasileira de Epidemiologia. 2018; 21 (Supl 1): 1-12.

19. Mundstock E, Toledo AO, Oliveira DG, Reimann P, Andrade FS, Veiga P et al. Resultados da primeira fase do Programa Esporte e Saúde em Canela, Rio Grande do Sul: avaliação do perfil nutricional. Scientia Medica. 2017; 27 (4): 1-6.

20. Pereira KAS, Nunes SEA, Miranda RSA, Horas AD, Almeida Júnior JP, Meireles AM et al. Fatores de risco e proteção contra doenças crônicas não transmissiveis entre adolescentes. Revista Brasileira da Promoção à Saúde. 2017; 30 (2): 205-212.

21. Anjos LA, Silveira WDB. Estado nutricional dos alunos da Rede Nacional de Ensino de Educação Infantil e Fundamental do Serviço Social do Comércio (Sesc), Brasil, 2012. Ciência \& Saúde Coletiva. 2017; 22 (5): 1725-1734.

22. Rostirolla LM. Obesidade infantil e sua influência na saúde de crianças e adolescentes. 2016. 29p. Monografia (Curso de Pós Graduação Gestão em Saúde Pública) - Universidade Alto Vale do Rio do Peixe - UNIARP. Caçador, Santa Catarina.

23. Borges LV, Carmo GV, Guerra MF, Lira CAB, Viana RB, Vancini RL et al. Prevalência de sobrepeso e obesidade em crianças e adolescentes de uma escola integral residentes em zona rural. Revista Uniandrade. 2017; 18 (3): 140-148.

24. Dias PC, Henriques P, Anjos LA, Burlandy L. Obesidade e políticas públicas: concepções e estratégias adotadas pelo governo brasileiro. Caderno de Saúde Pública. 2017; 33 (7): 1-12. 